\title{
INVERSE KINEMATICS FOR EDUCATIONAL ROBOT MOVER 4
}

\author{
Kaloyan Yankov \\ Faculty of Technics and Technologies, Trakia University, Bulgaria \\ 38 Graf Ignatiev str., Yambol, Bulgaria \\ e-mail: kaloyan.yankov@trakia-uni.bg
}

\begin{abstract}
The solution of inverse kinematics is a necessary condition to realize the control of a manipulation robot. The object of this work is to discuss some possible solutions of the inverse problem for the MOVER 4 robot. The kinematics equations are generated in analytic form with the CINDY program. These equations are the source to obtain the closed form solution. This is the approach that creates the fastest and most economical computer-memory algorithms. The disadvantage is that closed-form solutions are possible for a class of manipulation systems (MS) with a limited number of degrees of freedom. The second approach is more general and applies to MS where a closed-form solution is not possible. The goal function that must be minimized is the distance between the end-effector coordinates and the target point and the constraint is that decision must belong to the joint space coordinates. The task is solved applying the Lagrange multipliers. The disadvantage of the method is the probability to fall into a singular configuration where the Jacobian is zero. The third approach is the cyclic coordinate descent method. In this case, the multiparametric optimization is reduced to a one-parametric. The minimum of the goal function depends on only one degree of freedom at a time to move the end effector to the target point. This method is applicable to manipulation systems with an unlimited number of degrees of freedom. The disadvantage is that the solution is a local rather than a global minimum.
\end{abstract}

Keywords: robotics, inverse problem, Lagrange multipliers, optimization.

\section{INTRODUCTION}

The robot manipulation system (MS) is an open kinematic chain (OKC) of serially connected rigid objects, called links. The first link is considered stationary relative to a selected coordinate system. Two consecutive links are linked by kinematic joint. It performs the relative motion of the links and most often, for technological reasons, has one degree of mobility. Intermediate links belong to two kinematic joints. The terminal link has the role of end-effector (EE), performing the technological operation for which the robot is created. Each movement is realized by one rotation or translation. The kinematic joints are independent parameters that uniquely define the position of each point of MS in space. They are denoted by $n$-dimensional vector:

$$
q=\left[q_{1}, q_{2}, \ldots q_{n}\right]^{T}
$$

called configuration of the MS. The number of kinematic pairs determines the number of degrees of mobility of MS. In order to position and orient the EE in the space, 6 degrees of mobility is required. Due to the constructive constraints imposed on each joint and the possible presence of obstacles to be avoided, these 6 degrees may prove to be insufficient to realize the technological operations. The change the generalized coordinates $\boldsymbol{q}$ in accordance within the limits of their constraints defines an $n$-dimensional parallelepiped of permissible configurations: 


\section{IRTTIE}

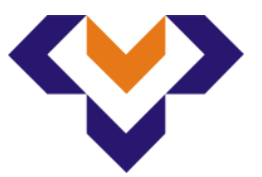
Ipplied Researroches in Technics, Technologies and Bduration Journal of the Faculty of Technics and Technologies, Trakia University https://sites.google.com/a/trakia-uni.bg/artte/

$$
Q_{0}=\left\{q: q_{i}^{\text {min }} \leq q_{i} \leq q_{i}^{\max }\right\}
$$

Two main tasks are important in creation and control of a manipulation robot..

\subsection{Direct Kinematics}

The coordinates of characteristic point $\boldsymbol{M}$ of EE are determined by the function $\boldsymbol{F}$ :

$$
M(x, y, z, \alpha, \beta, \gamma)=F(q)
$$

where: $\mathrm{F}$ - continuous nonlinear function;

$(x, y, z)$-- the position in Cartesian coordinates of the EE;

$(\alpha, \beta, \gamma)$ - the orientation in Euler angles of the EE.

Equation (3) defines the direct kinematics for open kinematics chain. The set

$$
D=\left\{M: M=F(q), q \in Q_{0}\right\}
$$

defines the robot's workspace. In this area must be located the tasks of the robot.

\subsection{Inverse Kinematics}

The direct kinematics is required to be able to calculate the actual position of the links and EE. However, in order to position and orient the EE at a desired point in the workspace, it is necessary to formulate and solve the so-called "Inverse Kinematics" (IK).

An initial configuration $\boldsymbol{q}_{0}$ is given:

$$
q_{0} \in Q, M_{0}\left(x_{0}, y_{0}, z_{0}, \alpha_{0}, \beta_{0}, \gamma_{0}\right)=F\left(q_{0}\right)
$$

The goal space coordinates of EE are:

$$
G\left(x_{G}, y_{G}, z_{G}, \alpha_{G}, \beta_{G}, \gamma_{G}\right) \in D
$$

and the generalized coordinates $q_{G}$ need to be computed

$$
F\left(q_{G}\right)=G
$$

looking for the inverse function $\boldsymbol{F}^{-1}$ for which it is satisfied:

$$
q_{G}=F^{-1}(G)
$$

Because of the non-linearity of $\boldsymbol{F}(\boldsymbol{q})$, finding the inverse function can be a difficult, even an unsolved task.

The purpose of this work is to present three different algorithms for solving the IK for the MOVER 4 robot. This will help to create a software for robot analysis and control, regardless of the accompanying CProg software whose code is closed. 


\section{ARTTIE}

Ipplied Researrches in Technics, Technologies and Bducition Journal of the Faculty of Technics and Technologies, Trakia University https:///ites.google.com/a/trakia-uni.bg/artte/

\section{EDUCATIONAL ROBOT MOVER 4}

The MOVER 4 robot of Commonplace Robotics GmbH [8] has four degrees of freedom and has a planar kinematic structure. Figure 1 is the robot scheme and in Table 1 are the kinematics parameter values. Three of the degrees of mobility provide the positioning, and the fourth orientates the EE relative to the $Z$ axis of the coordinate system associated with the base of the robot. The coordinates of any point in the kinematic chain, and in particular the $\mathrm{EE}$, can be derived from geometric considerations. In this case, a specialized language is used to describe the OKC [11]. The kinematic equations are generated using CINDY program [10]. In the syntax of this language, each joint is represented with one sentence ending with ";". The sentence consists of words separated by ",". The first word determines the type of kinematic pair: "R|RD|P" and its user-selected identifier. In the joint constraints are described. The following words in the sentence present the three-dimensional transformations (rotation or translation) relative to the previous joint. The kinematic model in terms of this language is shown in Figure 2.

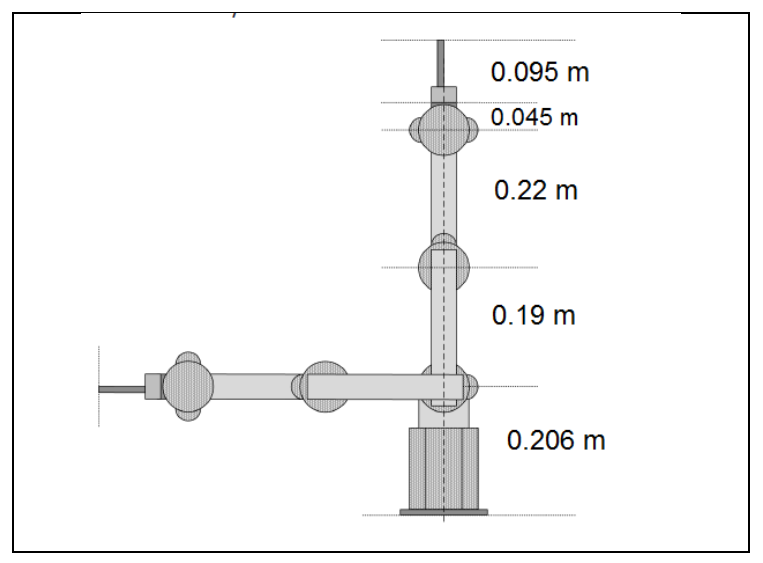

Figure 1. Robot MOVER 4

Table 1. Kinematic parameters

\begin{tabular}{|c|c|c|c|c|}
\hline $\begin{array}{c}\text { Joint } \\
\text { number } \\
\boldsymbol{n}\end{array}$ & $\begin{array}{c}\text { Constraints } \\
\boldsymbol{\theta n}[\mathrm{DEG}]\end{array}$ & $\begin{array}{c}\text { Length } \\
\boldsymbol{L n}[\mathrm{m}]\end{array}$ & $\begin{array}{c}\text { twist angle } \\
\boldsymbol{a n}[\mathrm{DEG}]\end{array}$ & $\begin{array}{c}\text { Offset } \\
\boldsymbol{S n}[\mathrm{m}]\end{array}$ \\
\hline 1 & $-150,150$ & 0 & 90 & 0.206 \\
\hline 2 & $-50,65$ & 0.19 & 0 & 0 \\
\hline 3 & $-110,140$ & 0.22 & 0 & 0 \\
\hline 4 & $-140,135$ & 0.095 & 0 & 0 \\
\hline
\end{tabular}

RDq1(-150,150), RAD(90),TS(S1);

RDq2(-50,65), TA(L2);

RDq3(-110,140), TA(L3);

RDq4(-140,135), TA(L4);

Figure 2. Description of the MOVER 4 


\section{IRTITIE

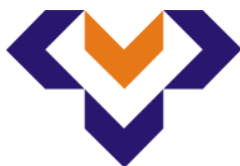 \\ Ipplied Resseirlores in Technics, Technologies and Educration \\ Journal of the Faculty of Technics and Technologies, Trakia University https:///ites.google.com/a/trakia-uni.bg/artte/}

The generated equations for the $(X, Y, Z)$-coordinates of the $E E$ are:

$$
\mid \begin{aligned}
& X=\cos (q 1) \cdot(\cos (q 2) \cdot L 2+\cos (q 2+q 3) \cdot L 3+\cos (q 2+q 3+q 4) \cdot L 4) \\
& Y=\sin (q 1) \cdot(\cos (q 2) \cdot L 2+\cos (q 2+q 3) \cdot L 3+\cos (q 2+q 3+q 4) \cdot L 4) \\
& Z=S 1+\sin (q 2) \cdot L 2+\sin (q 2+q 3) \cdot L 3+\sin (q 2+q 3+q 4) \cdot L 4
\end{aligned}
$$

This is the output system from which the solutions of the inverse problem must be obtained.

\section{INVERSE KINEMATICS}

In the Inverse kinematics, the coordinates of the goal point are known, as well as the desired orientation vector of the EE by the Euler angles. The values of the generalized coordinates $\left[q_{1}, q_{2}, q_{3}, q_{4}\right]$ with which the target point can be reached must be calculated. The fact that the system (8) is transcendent leads to serious computational problems. From the moment that robotics has been the subject of scientific research, solving the reverse is the eternal theme.

\subsection{Geometric solution}

An algebraic solution of the trigonometric equations (8) is difficult, maybe impossible. An important fact is that the kinematic circuit is planar. Therefore, a suitable approach is to seek a solution based on geometric relationships in the plane of the MS.

The goal point is $\mathbf{G}\left(\boldsymbol{G}_{X}, \boldsymbol{G}_{\boldsymbol{Y}}, \boldsymbol{G}_{Z,}, \boldsymbol{Y}\right)$. The first joint of the robot is rotation around an axis $Z$ of a coordinate system attached to its base (Figure 3). Because the kinematic chain is planar, the dependencies will be viewed in the plane defined by the Z-axis and the target point $G$. The first angle that is easily calculated is the rotation of the first link around the Z-axis:

$$
q_{1}=a \tan \left(\frac{G_{Y}}{G_{X}}\right)
$$

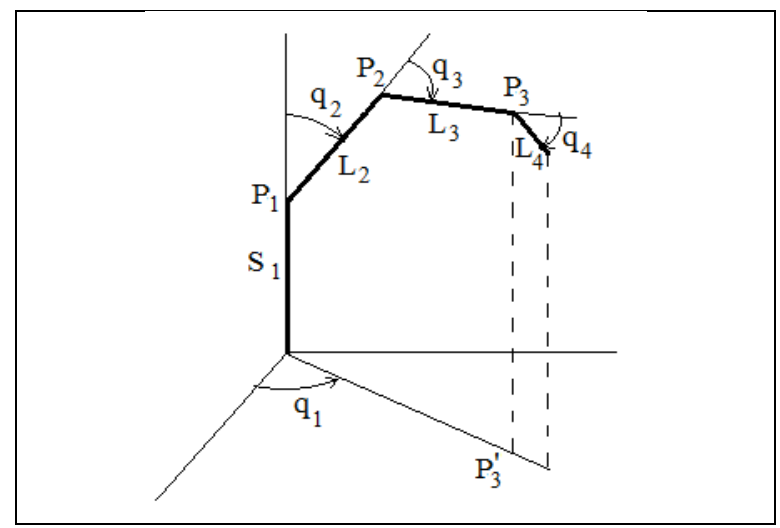

Figure 3. Kinematics model of MOVER 4

The approach orientation is given with only one angle $\boldsymbol{V}$, toward to the $Z$ axis (Figure 4). It helps to determine the coordinates of the beginning of the $E E$ - point $P_{3}\left(P_{3 X}, P_{3 Y}, P_{3 Z}\right)$ : 


\section{ARITIE}

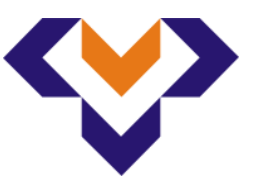
Ipplied Researrches in Technics, Technologies and Bducition Journal of the Faculty of Technics and Technologies, Trakia University https://sites.google.com/a/trakia-uni.bg/artte/

$$
\left\|\begin{array}{c}
P_{3 X} \\
P_{3 Y} \\
P_{3 Z}
\end{array}\right\|=\left\|\begin{array}{c}
G_{X}+L_{4} \cdot \sin (\gamma) \cdot \cos \left(q_{1}\right) \\
G_{Y}+L_{4} \cdot \sin (\gamma) \cdot \sin \left(q_{1}\right) \\
G_{Z}+L_{4} \cdot \cos (\gamma)
\end{array}\right\|
$$

For triangle $\mathrm{P}_{1} \mathrm{P}_{2} \mathrm{P}_{3}$ (Figure 5) a cosine theorem is applied:

$$
\left(P_{1} P_{3}\right)^{2}=L_{2}^{2}+L_{3}^{2}-2 \cdot L_{2} \cdot L_{3} \cdot \cos \left(\pi-q_{3}\right)
$$

From this expression is obtained:

$$
\cos \left(q_{3}\right)=\frac{\left(P_{1} P_{3}\right)^{2}-\left(L_{2}^{2}+L_{3}^{2}\right)}{2 \cdot L_{2} \cdot L_{3}}
$$

From Pythagorean theorem in $\triangle \mathrm{P}_{1} \mathrm{P}_{5} \mathrm{P}_{3}$ :

$$
\left(P_{1} P_{3}\right)^{2}=P_{3 X}^{2}+P_{3 Y}^{2}+\left(P_{3 Z}-S_{1}\right)^{2}
$$

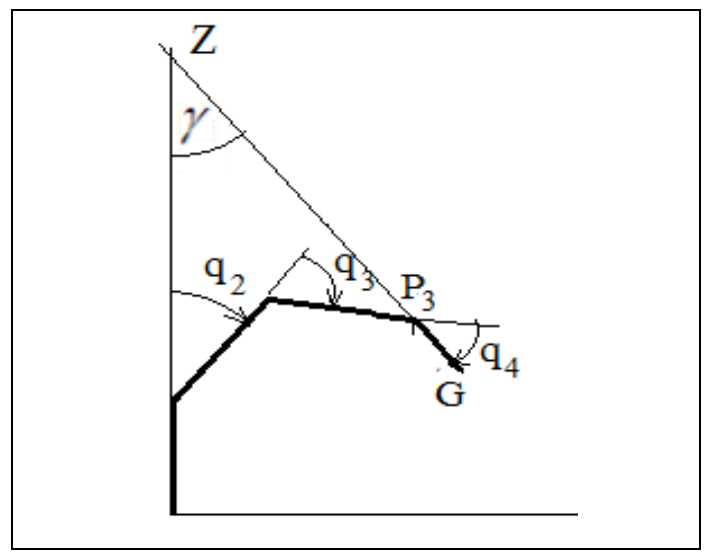

Figure 4. Plane of the MS

And for angle $\boldsymbol{q}_{3}$ is obtained:

$$
q_{3}=\arccos \frac{P_{3 X}^{2}+P_{3 Y}^{2}+\left(P_{3 Z}-S_{1}\right)^{2}-\left(L_{2}^{2}+L_{3}^{2}\right)}{2 \cdot L_{2} \cdot L_{3}}
$$

Angle $\boldsymbol{q}_{\mathbf{2}}$ is calculated from geometric dependencies on Figure 5 .

$$
q_{2}=\frac{\pi}{2}-(\varphi+\delta)
$$

In $\triangle \mathrm{P}_{1} \mathrm{P}_{4} \mathrm{P}_{3}$ angle $\boldsymbol{\varphi}$ is:

$$
\varphi=a \tan \frac{L_{3}^{2} \cdot \sin \left(q_{3}\right)}{L_{2}+L_{3} \cdot \cos \left(q_{3}\right)}
$$

IRTIIE Vol. 5, No. 3, 2017 ISSN 1314-8788 (print), ISSN 1314-8796 (online), doi: 10.15547/artte.2017.03.009 


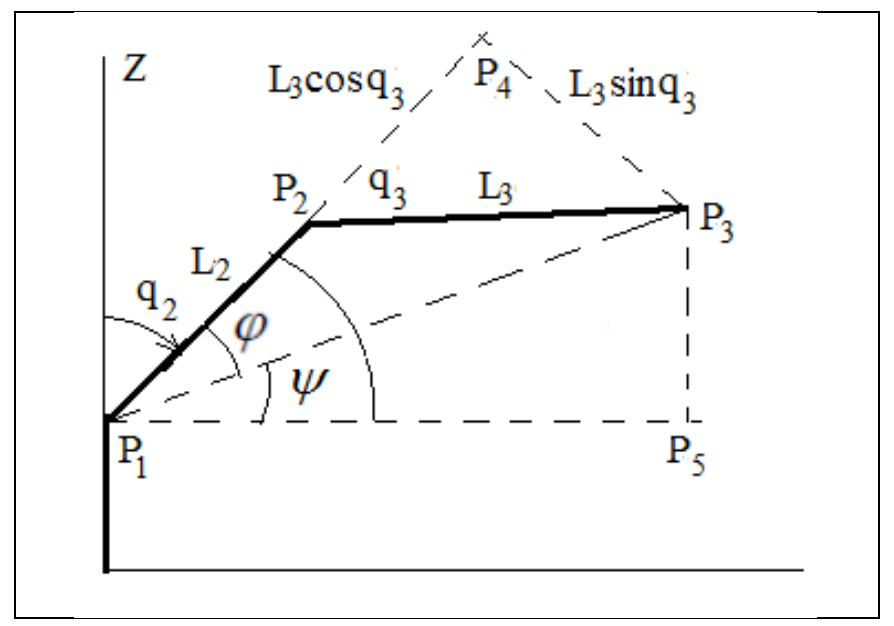

Figure 5. Relationship for angles $\boldsymbol{q}_{2} \mathrm{n} \boldsymbol{q}_{3}$

In $\Delta \mathrm{P}_{1} \mathrm{P}_{5} \mathrm{P}_{3}$ - angle $\boldsymbol{\psi}$ :

$$
\psi=a \tan \frac{P_{3 Z}-S_{1}}{\sqrt{P_{3 X}^{2}+P_{3 Y}^{2}}}
$$

For $\boldsymbol{q}_{\mathbf{2}}$ the explicit dependence is obtained:

$$
q_{2}=\frac{\pi}{2}-\frac{L_{3}^{2} \cdot \sin \left(q_{3}\right)}{L_{2}+L_{3} \cdot \cos \left(q_{3}\right)}-a \tan \frac{P_{3 Z}-S_{1}}{\sqrt{P_{3 X}^{2}+P_{3 Y}^{2}}}
$$

It remains to find the last angle - $\boldsymbol{q}_{4}$, which defines the desired angle of approach $\boldsymbol{y}$ to the target point. Figure 6 shows the dependence:

From that follows, that

$$
q_{2}+q_{3}+q_{4}+\gamma=\pi
$$

$$
q_{4}=\pi-\left(q_{2}+q_{3}+\gamma\right)
$$

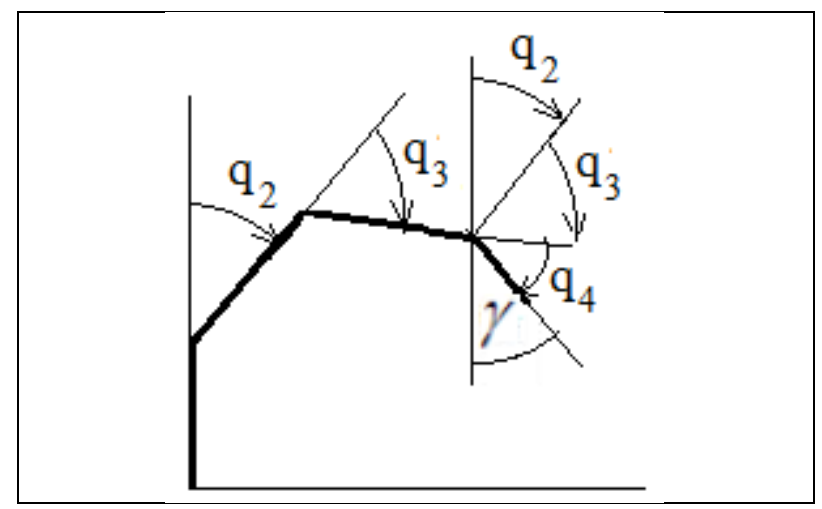

Figure 6. Approach angle $\boldsymbol{q}_{4}$

IRTIIE Vol. 5, No. 3, 2017 ISSN 1314-8788 (print), ISSN 1314-8796 (online), doi: 10.15547/artte.2017.03.009 


\section{ART'TE \\ Ipplied Researreches in Technics, Technologies and Bdurition \\ Journal of the Faculty of Technics and Technologies, Trakia University https:///ites.google.com/a/trakia-uni.bg/artte/}

Finally, the algorithm for calculating the generalized coordinates is:

Algorithm 1. Closed form solution of inverse kinematics

Input data: $\mathrm{S}_{1}, \mathrm{~L}_{2}, \mathrm{~L}_{3}, \mathrm{~L}_{4}, \mathrm{G}\left(\mathrm{G}_{\mathrm{X}}, \mathrm{G}_{\mathrm{Y}}, \mathrm{G}_{\mathrm{Z}}, \mathrm{Y}\right)$

\section{Calculations:}

1. $q_{1}=a \tan \left(\frac{G_{Y}}{G_{X}}\right)$

2. $P_{3 X}=G_{X}+L_{4} \cdot \sin (\gamma) \cdot \cos \left(q_{1}\right)$

$P_{3 Y}=G_{Y}+L_{4} \cdot \sin (\gamma) \cdot \sin \left(q_{1}\right)$

$P_{3 Z}=G_{Z}+L_{4} \cdot \cos (\gamma)$

3. $q_{3}=\arccos \frac{P_{3 X}^{2}+P_{3 Y}^{2}+\left(P_{3 Z}-S_{1}\right)^{2}-\left(L_{2}^{2}+L_{3}^{2}\right)}{2 \cdot L_{2} \cdot L_{3}}$

4. $q_{2}=\frac{\pi}{2}-\frac{L_{3}^{2} \cdot \sin \left(q_{3}\right)}{L_{2}+L_{3} \cdot \cos \left(q_{3}\right)}-a \tan \frac{P_{3 Z}-S_{1}}{\sqrt{P_{3 X}^{2}+P_{3 Y}^{2}}}$

5. $q_{4}=\pi-\left(q_{2}+q_{3}+\gamma\right)$

A short, clear, accurate linear algorithm.

\subsection{Lagrange multipliers}

Let the coordinates $\boldsymbol{M}_{\boldsymbol{0}}$ of the end-effector at initial configuration $\boldsymbol{q}_{\boldsymbol{0}}$ in accordance with equation (3) are:

$$
M_{0}=F\left(q_{0}\right)
$$

Goal point $\boldsymbol{G}$ is reached with configuration $\boldsymbol{q}_{\boldsymbol{G}}$ :

$$
G=F\left(q_{G}\right)
$$

Assuming that point $\boldsymbol{G}$ is in the neighbourhood of the starting point $\boldsymbol{M}_{\boldsymbol{0}}$, then the target configuration can be presented in a Taylor series:

$$
G=F\left(q_{G}\right) \approx F\left(q_{0}\right)+\frac{\partial F\left(q_{0}\right)}{\partial q}\left(q-q_{0}\right)+\frac{1}{2 !} \frac{\partial^{2} F\left(q_{0}\right)}{\partial q^{2}}\left(q-q_{0}\right)^{2}+\frac{1}{3 !} \frac{\partial^{3} F\left(q_{0}\right)}{\partial q^{3}}\left(q-q_{0}\right)^{3}+\ldots
$$

Taking two initial terms, the next polynomial is formed

$$
G=F_{0}+J_{0}\left(q_{G}-q_{0}\right)
$$

Where $\boldsymbol{J}_{0}$ is the Jacobian matrix: 


\section{ARITIE}

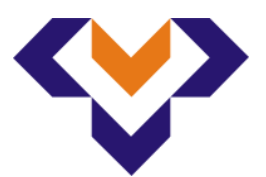
Ipplied Researroches in Technics, Technologies and Bduration Journal of the Faculty of Technics and Technologies, Trakia University https://sites.google.com/a/trakia-uni.bg/artte/

$$
J_{0}=\left\|\begin{array}{llll}
\frac{\partial F_{1}}{\partial q_{1}} & \frac{\partial F_{1}}{\partial q_{2}} & \cdots & \frac{\partial F_{1}}{\partial q_{n}} \\
\frac{\partial F_{2}}{\partial q_{1}} & \frac{\partial F_{2}}{\partial q_{2}} & \cdots & \frac{\partial F_{2}}{\partial q_{n}} \\
\frac{\partial \dot{F}_{m}}{\partial q_{1}} & \frac{\partial \dot{F}_{m}}{\partial q_{2}} & & \frac{\partial \dot{F}_{m}}{\partial q_{n}}
\end{array}\right\|
$$

The solution of the Eq.(17) to the target configuration is:

$$
q_{G}=J_{0}^{-1}\left(G-M_{0}\right)+I \cdot q_{0}
$$

The inverse matrix $J_{0}^{-1}$ does not always exist. The system (19) has a solution if

$$
\operatorname{rank}\left(J_{0}\right)=n
$$

Manipulation configurations where the rank of Jacobian matrix is less than its maximum value are called singular. They a serious problem, and efforts to avoid or get out of them are still relevant $[5,6,7]$.

In the present work, one approach will be considered that solves the problem but does not guarantee accuracy in the meaning of Algorithm 1. The method of the Lagrange multipliers will be applied $[1,4]$. This approach is a strategy for finding the minima of a function subject to equality constraints. The goal function is to minimize the Euclidean norm $\boldsymbol{S}\left(\boldsymbol{q}_{0}, \boldsymbol{q}_{\mathrm{G}}\right)$ between the EE and the target point:

$$
S\left(q_{0}, q_{G}\right)=\left\|q_{G}-q_{0}\right\|^{T} \cdot\left\|q_{G}-q_{0}\right\| \rightarrow \min
$$

The limiting condition is $\mathrm{Eq}(17)$ :

$$
Y\left(q_{0}, q_{G}\right)=J_{0}\left(q_{G}-q_{0}\right)-\left(G-M_{0}\right)=0
$$

To find the minimum of $\boldsymbol{S}\left(\boldsymbol{q}_{0}, \boldsymbol{q}_{\mathrm{G}}\right)$, the Lagrange function must be solved:

$$
\mid \begin{aligned}
& \nabla S\left(q_{0}, q_{G}\right)=\lambda^{T} . \nabla Y\left(q_{0}, q_{G}\right) \\
& Y\left(q_{0}, q_{G}\right)=0
\end{aligned}
$$

where $\lambda$ is Lagrange multiplier .

After replacing goal function (20) in system (22):

$$
\left\|q_{G}-q_{0}\right\|^{T} \cdot\left\|q_{G}-q_{0}\right\|=\lambda^{T}\left[J_{0}\left(q_{G}-q_{0}\right)-\left(G-M_{0}\right)\right]
$$

and differentiating the last expression, we get the expression for $\boldsymbol{q}_{\mathrm{G}}$ :

$$
q_{G}=q_{0}+J_{0}{ }^{T} \lambda
$$

It is replaced in (21) to obtain the value of the multiplier $\lambda$ :

IRITIE Vol. 5, No. 3, 2017 ISSN 1314-8788 (print), ISSN 1314-8796 (online), doi: 10.15547/artte.2017.03.009 


\section{ARITIE} Ipplied Researreches in Technics, Technologies and Eductation Journal of the Faculty of Technics and Technologies, Trakia University https://sites.google.com/a/trakia-uni.bg/artte/

$$
\lambda=\left(J_{0} \cdot J^{T}\right)^{-1} \cdot\left(G-M_{0}\right)
$$

It can be seen that, $\operatorname{rank}\left(J_{0} \cdot J^{T}\right)^{-1}=m$, therefore, the matrix $\left(J_{0} \cdot J^{T}\right)^{-1}$ is computable. The target vector of the generalized coordinates already has value:

$$
q_{G}=q_{0}+J_{0}^{T}\left(J_{0} \cdot J^{T}\right)^{-1} \cdot\left(G-M_{0}\right)
$$

The matrix $J^{\#}=J_{0}^{T}\left(J_{0} \cdot J^{T}\right)^{-1}$ is called Moore-Penrose pseudoinverse with the following important properties:

$\checkmark \quad J^{*}$ always exists and is unique.

$\checkmark \quad J J^{\#}=1$

$\checkmark \quad$ If the Jacobian $J$ has no full rank, $J^{\#}$ can be computed with a numeric algorithm.

It is obvious that this algorithm is hard to implement, requires many matrix operations, does not give a precise solution, and has a serious drawback - the target point must be in the vicinity of the EE initial position - this is the condition to use Taylor series. An advantage is that it can be used for manipulation systems where a closed form solution is impossible, as well as for MS with more than 6 degrees of freedom [2].

\subsection{Cyclic coordinate descent method (CCD)}

\subsubsection{Formulation of the method}

A goal function $\boldsymbol{\Phi}(q)$ between the initial $M_{0}=F\left(q_{0}\right)$ and the target position $G$ of the EE is formed :

$$
\Phi(q)=\left\|G-F\left(q_{0}\right)\right\|
$$

By successively performing approximate cyclic minimization of $\Phi(q)$ along each parameter $\boldsymbol{q}_{i}$, the minimum of the target function is obtained:

Step 1.

$$
\begin{array}{ll}
q_{2}, q_{3}, \ldots, q_{n-1} q_{n} & \text { fixed ; } \\
\frac{\partial \Phi(q)}{\partial q_{1}}=0 & q_{1}^{(1)} \text { is defined }
\end{array}
$$

Step 2. $q_{1}^{(1)}, q_{3}, \ldots, q_{n-1} q_{n} \quad$ fixed ;

$$
\frac{\partial \Phi(q)}{\partial q_{2}}=0 \quad q_{2}^{(1)} \text { is defined ; }
$$

Step n. $\quad q_{1}^{(1)}, q_{2}^{(1)}, \ldots, q_{n-2}^{(1)}, q_{n-1}^{(1)} \quad$ fixed ;

$$
\frac{\partial \Phi(q)}{\partial q_{n}}=0 \quad \quad q_{n}^{(1)} \text { is defined; }
$$




\section{IRTTIE

When looking at the $\boldsymbol{i}$-th coordinate $\boldsymbol{q}_{\boldsymbol{i}}$, the remaining $(\boldsymbol{i}-\mathbf{1})$ joints are considered unchangeable. Each iteration consists of $\boldsymbol{n}$ steps, equal to the number of joints. The values of $\boldsymbol{\Phi}(\boldsymbol{q})$ obtained in successive $\boldsymbol{k}$ iterations form the sequence:

$$
\Phi_{0}\left(q^{(0)}\right) \geq \Phi_{1}\left(q^{(1)}\right) \geq \Phi_{2}\left(q^{(2)}\right) \geq \ldots \geq \Phi_{k}\left(q^{(k)}\right) \geq 0
$$

The sequence is monotonically decreasing and is bounded below, therefore it converges to a positive number $\boldsymbol{\varepsilon}$. This is a Euclidean distance convergence. Because

$$
\Phi_{m}\left(q^{(m)}\right) \underset{m \rightarrow k}{\longrightarrow} 0
$$

from distance convergence follows coordinate convergence [3]. If:

$$
\Phi_{k}(q) \leq \varepsilon
$$

and $\varepsilon$ is a small neighbourhood of the goal point, then the task is solved with accuracy $\varepsilon$. On the contrary, if for two consecutive iterations the function $\boldsymbol{\Phi ( q )}$ does not change and (27) is not fulfilled, ie:

$$
\Phi_{k}(q)>\varepsilon, \Phi_{k-1}(q)=\Phi_{k}(q)
$$

the solution is not a global but a local minimum. Falling to a local minimum is a serious disadvantage of the method and one of the main reasons why it is not used. Another inconvenience is the need to calculate partial derivatives - this creates more problems than facilities.

\subsubsection{Modification of CCD}

Instead of using partial derivatives to minimize the target function, geometric considerations may be used to search for a minimum between a goal point and a EE [9]. When rotating a corresponding kinematic pair, EE describes a circle with a center on the axis of the joint selected for optimization. A minimum distance between $E E$ and $G$ will have when the $E E$ belongs to the line formed by the point of rotation of the joint and the target point $\mathbf{G}$ (Figure 7).

Because the kinematic structure introduces a limit on the orientation only to the $Z$ axis, it is necessary to select an intermediate target point $\boldsymbol{G}^{*}$, which is calculated by (10). $\boldsymbol{G}^{*}$ is the target point for the end of the third unit (starting point of EE):

$$
\left\|\begin{array}{c}
G_{X}^{*} \\
G_{Y}^{*} \\
G_{Z}^{*}
\end{array}\right\|=\left\|\begin{array}{c}
G_{X}+L_{4} \cdot \sin (\gamma) \cdot \cos \left(q_{1}\right) \\
G_{Y}+L_{4} \cdot \sin (\gamma) \cdot \sin \left(q_{1}\right) \\
G_{Z}+L_{4} \cdot \cos (\gamma)
\end{array}\right\|
$$




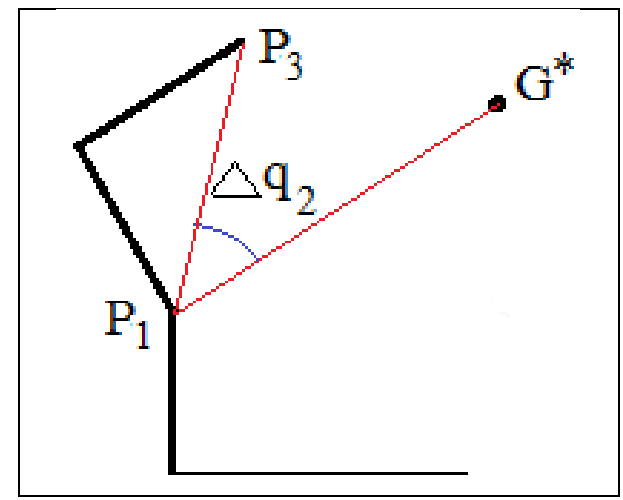

Figure 7. Minimization of $\boldsymbol{q}_{2}$

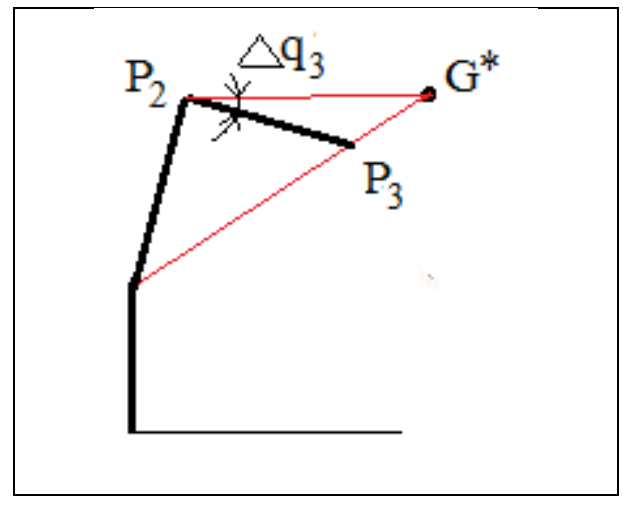

Figure 8. Minimization of $\boldsymbol{q}_{3}$

Step 1. Calculation of joint $\mathbf{q}_{2}$.

Vector $\vec{P}_{1} P_{3}$ is:

$$
\overrightarrow{P_{1} P_{3}}=\left\|\begin{array}{c}
\cos \left(q_{1}\right) \cdot\left[\cos \left(q_{2}\right) \cdot L 2+\cos \left(q_{2}+q_{3}\right) \cdot L 3\right] \\
\sin \left(q_{1}\right) \cdot\left[\cos \left(q_{2}\right) \cdot L 2+\cos \left(q_{2}+q_{3}\right) \cdot L 3\right] \\
\sin \left(q_{2}\right) \cdot L 2+\sin \left(q_{2}+q_{3}\right) \cdot L 3
\end{array}\right\| \cdot\left\|\begin{array}{l}
\vec{i} \\
\vec{j} \\
\vec{k}
\end{array}\right\|
$$

Vector $\overrightarrow{P_{1} G^{*}}$ :

$$
\overrightarrow{P_{1} G^{*}}=\left\|\begin{array}{c}
G_{X}+L_{4} \cdot \sin (\gamma) \cdot \cos \left(q_{1}\right)-\cos \left(q_{1}\right) \cdot\left[\cos \left(q_{2}\right) \cdot L 2+\cos \left(q_{2}+q_{3}\right) \cdot L 3\right] \\
G_{Y}+L_{4} \cdot \sin (\gamma) \cdot \sin \left(q_{1}\right)-\sin \left(q_{1}\right) \cdot\left[\cos \left(q_{2}\right) \cdot L 2+\cos \left(q_{2}+q_{3}\right) \cdot L 3\right] \\
G_{Z}+L_{4} \cdot \cos (\gamma)-S 1-\left[\sin \left(q_{2}\right) \cdot L 2+\sin \left(q_{2}+q_{3}\right) \cdot L 3\right]
\end{array}\right\| \cdot\left\|\begin{array}{c}
\vec{i} \\
\vec{j} \\
\vec{k}
\end{array}\right\|
$$

The angle between the two vectors is $\Delta \mathrm{q}_{2}$ :

$$
\cos \left(\Delta q_{2}\right)=\frac{\overrightarrow{P_{1} P_{3}} \cdot \overrightarrow{P_{1} G^{*}}}{\left|\overrightarrow{P_{1} P_{3}}\right| \cdot\left|\overrightarrow{P_{1} G^{*}}\right|}
$$

For angle $\Delta q_{2}$ is obtained:

$$
\Delta q_{2}=\arccos \left(\frac{\overrightarrow{P_{1} P_{3}} \cdot \overrightarrow{P_{1} G^{*}}}{\left|\overrightarrow{P_{1} P_{3}}\right|\left|\overrightarrow{P_{1} G^{*}}\right|}\right)
$$

The required value for reaching a minimum distance to the target point is:

$$
\mathrm{q}_{2}=\mid \begin{aligned}
& q_{2}^{\min }, \text { if }: q_{2}+\Delta q_{2}<q_{2}^{\min } \\
& q_{2}^{\max }, \text { if }: q_{2}+\Delta q_{2}>q_{2}^{\max } \\
& q_{2}+\Delta q_{2}, \text { if }: q_{2}^{\min }<\left(q_{2}+\Delta q_{2}\right)<q_{2}^{\max }
\end{aligned}
$$

IRTIIE Vol. 5, No. 3, 2017 ISSN 1314-8788 (print), ISSN 1314-8796 (online), doi: 10.15547/artte.2017.03.009 


\section{IRTTIE

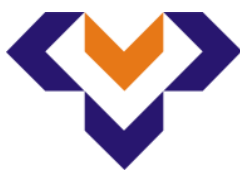

Ipplied Resseirlores in Technics, Technologies and Educration

Journal of the Faculty of Technics and Technologies, Trakia University https://sites.google.com/a/trakia-uni.bg/artte/

\section{Step 2. Calculation of joint $\boldsymbol{q}_{3}$.}

Vector $\overrightarrow{P_{2} P_{3}}$ is obtained from Figure 8:

Vector $\overrightarrow{P_{2} G^{*}}$ :

$$
\overrightarrow{P_{2} P_{3}}=\left\|\begin{array}{c|c}
\cos \left(q_{1}\right) \cdot \cos \left(q_{2}+q_{3}\right) \cdot L 3 \\
\sin \left(q_{1}\right) \cdot \cos \left(q_{2}+q_{3}\right) \cdot L 3 \\
\sin \left(q_{2}+q_{3}\right) \cdot L 3
\end{array}\right\| \cdot\left\|\begin{array}{c}
\vec{i} \\
\vec{j} \\
\vec{k}
\end{array}\right\|
$$

$$
\overrightarrow{P_{2} G^{*}}=\left\|\begin{array}{c}
G_{X}+L_{4} \cdot \sin (\gamma) \cdot \cos \left(q_{1}\right)-\cos \left(q_{1}\right) \cdot \cos \left(q_{2}+q_{3}\right) \cdot L 3 \\
G_{Y}+L_{4} \cdot \sin (\gamma) \cdot \sin \left(q_{1}\right)-\sin \left(q_{1}\right) \cdot \cos \left(q_{2}+q_{3}\right) \cdot L 3 \\
G_{Z}+L_{4} \cdot \cos (\gamma)-S 1-\sin \left(q_{2}+q_{3}\right) \cdot L 3
\end{array}\right\| \cdot\left\|\begin{array}{l}
\vec{i} \\
\vec{j} \\
\vec{k}
\end{array}\right\|
$$

The angle between the two vectors is $\Delta \mathrm{q}_{3}$ :

$$
\cos \left(\Delta q_{3}\right)=\frac{\overrightarrow{P_{2} P_{3}} \cdot \overrightarrow{P_{2} G^{*}}}{\left|\overrightarrow{P_{2} P_{3}}\right| \cdot\left|\overrightarrow{P_{2} G^{*}}\right|}
$$

And the angle $\Delta \mathrm{q}_{3}$ is:

$$
\Delta q_{3}=\arccos \left(\frac{\overrightarrow{P_{2} P_{3}} \cdot \overrightarrow{P_{2} G^{*}}}{\left|\overrightarrow{P_{2} P_{3}}\right|\left|\overrightarrow{P_{2} G^{*}}\right|}\right)
$$

The minimum distance to the target point with respect to the constraints is:

$$
\mathrm{q}_{3}=\mid \begin{aligned}
& q_{3}^{\min }, \text { if }: q_{3}+\Delta q_{3}<q_{3}^{\min } \\
& q_{3}^{\max }, \text { if }: q_{3}+\Delta q_{3}>q_{3}^{\max } \\
& q_{3}+\Delta q_{3}, \text { if }: q_{3}^{\min }<\left(q_{3}+\Delta q_{3}\right)<q_{3}^{\max }
\end{aligned}
$$

\section{Step 3. Verification of solution.}

If the target point is not reached but the process is converging, go to Step 1.

If the target point is reached in a neighborhood or the process is not converging - END.

The basic quality of this method is that it reduces the multiparametric optimization to a single one. The resulting decision is approximate - it is necessary to set a neighborhood $\boldsymbol{\varepsilon}$ as a goal criterion. The convergence of the CCD is slightly dependent on the discrepancy between the $\mathrm{EE}$ and the target position. Another important advantage is that it allows a free choice of the coordinate to be optimized. Depending on the specific conditions of movement, the degree of participation of the each joint can be varied, allowing optimization of the control.

Major disadvantages that prevent its wide application are that the algorithm may stall at a non-optimal point and the convergence rate is not good for points belonging on the periphery of the workspace. 


\section{ARTTIE $Y$}

Ipplied Resseirlores in Technics, Technologies and Educration

Journal of the Faculty of Technics and Technologies, Trakia University https://sites.google.com/a/trakia-uni.bg/artte/

\section{CONCLUSIONS}

In the present work, three methods for solving the inverse kinematics of the robot MOVER 4 are discussed. The methods have been modified according to the specific kinematic structure of the manipulation system, and the goal is to obtain computationally lightweight algorithms.

The closed form solution, based on the planar structure of the MS and derived from geometrical considerations, is the fastest for computing. A disadvantage may be the uncoordinated change of joints when moving to a target position.

Linearization in the vicinity of the work point in Taylor series is a versatile approach applicable to arbitrary structures. The disadvantage is the slow convergence and the likelihood of falling into a singular configuration. This algorithm is the heaviest in computing relation to the other two. It is suitable for trajectory robot control.

The coordinate descent method is a natural approach to finding a minimum Euclidean distance. Easy to compute, it allows a change in the order of calculation of the generalized coordinates, which is an advantage in the implementation of collision avoidance algorithms. As a minor drawback can be indicated the different number of iterations for different points in the workspace, and as very large - the likelihood of falling into a local minimum.

\section{ACKNOWLEDGEMENTS}

This study was supported by Grant 3ФTT/30.04.2015 "Identification and Simulation of Second-order Dynamic Models" from the Faculty of Technics and Technologies, Trakia University - Yambol, Bulgaria.

\section{REFERENCES}

[1] Borwein J.M. (1999). Convex Analysis and Nonlinear Optimization. Theory and Examples. Simon Fraser University, Burnaby, B.C., Canada V5A 1 S6.

[2] Konstantinov M., Markov M., Nenchev D. (1981). Kinematic Control of Redundant Manipulators. Proc. 11 ${ }^{\text {th }}$ ISIR, Tokyo, Japan, pp.561-568.

[3] Lusternik I.A., Sobolev V.I. (1965). Elements of functional analysis. Moskva, "Nauka".

[4] Manolov S., Deneva A. (1973). Mathematics. Vol. 2. Sofia, TEchnika, p.426.

[5] Nenchev D. (1983). Oriented movements of robots with active control. Ph.D.Thesis. Technical University, Sofia.

[6] Nielsen L.,Hagender P.(1990).Controllability Issues in Robot Near Singular Configurations. $2^{\text {nd }}$ Workshop on Advances in Robot Kinematics. Sept.10-12. Linz, Austria.

[7] Senft V., Hircinger G., (1995). Redunant Motions of non-redundant Robots - A New Approach to Singularity Treatment. Proc. IEEE Int. Conf. on Robotics and Automation. vol.2,1553 - 1559.

[8] User Guide Mover4 Version 2015/03 (SW V902-08-008, HWE 2MV23 HWM V05 DOC V14). Commonplace Robotics GmbH. Germany.

[9] Yankov K. (1991). Kinematics Simulation in robotics. Ph.D.Thesis. Technical University, Sofia.

[10] Yankov K. (1992). Computer Simulation of Industrial Robots. Proc.Int.Conf. ACMBUL'92, okt.4-8, st.Konstantine resort, Varna, Bulgaria, pp.33.1-33.8.

[11] Yankov K. (2002). Application-Oriented Language for Describing Open Kinematic Chains, Proc. 16-th Int.Conf. "Systems for Automation of Engineering and Research" SAER'2002, St.Konstantin resort, Varna, Bulgaria, 20-22 sept. pp.95-99. 